\title{
Relación entre la motivación intrínseca y la experiencia de flujo (flow) en una muestra
} de corredores costarricenses

\section{Diana Garita-Campos, Flavia Marcori y Rafael Román}

\section{Universidad de Iberoamérica}

\section{Resumen:}

Por medio de la aplicación de un cuestionario a una muestra de corredores de 42k y menos, se investiga la relación entre la motivación y el estado de flujo posterior a una competición. Se encontró una relación positiva entre la motivación y algunas de las dimensiones del flow en la muestra consultada.

Palabras clave: Corredores, Motivación, Experiencia de flujo, Psicología del deporte

\section{Introducción}

Las carreras de atletismo se han convertido en una forma popular de realizar actividad física entre los costarricenses. Según estadísticas de la Federación Costarricense de Atletismo, antes del año 2011 se realizaban aproximadamente 50 carreras y para ese mismo año la cantidad registradas aumentó a aproximadamente 84 carreras solamente en ruta. Este incremento también se ve reflejado en la cantidad de participantes, en donde las carreras más representativas del país registraban al inicio entre 500 y 1000 corredores y ahora rondan entre los 3000 y 5000 corredores (La Nación, 2011)

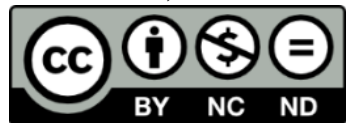


Dentro de las muy variadas sensaciones y emociones que se pueden experimentar antes, durante y después de una carrera de atletismo, una de ellas es el flow, la cual ha sido descrita como un "estado de conciencia en el que uno llega a estar totalmente absorto por la actividad que está realizando, hasta alcanzar la exclusión de todo pensamiento o emoción" (Fernández, Godoy-Izquierdo, Jaenes, Bohórquez y Vélez, 2015). El estudio del flow en el deporte se caracteriza por estar relacionado con otras mediciones que pueden influir en el desarrollo competitivo de los atletas, como la motivación intrínseca (Koehn, Donald y Paramei, 2018).

Tomando en cuenta lo antes mencionado y considerando que las carreras de atletismo son cada vez más populares en Costa Rica, se presenta un vacío en el conocimiento acerca de la relación entre la motivación intrínseca, el estado de flow y la actividad física de corredores que participan en distancias de $42 \mathrm{k}$ y menores.

Algunas investigaciones ya han estudiado el tema y arrojan algunas relaciones importantes de considerar. En la ciudad de Barcelona, López, Torregrosa y Roca (2007), evaluaron y compararon las dimensiones de "flow", ansiedad y estado emocional de las mejores y las peores experiencias de rendimiento deportivo en nadadores de élite. Para ello utilizaron entrevistas individuales, en las que participaron 10 personas; 7 hombres y 3 mujeres, quienes relataban los detalles de lo que cada uno consideraba su mejor y peor experiencia en este campo. Entre los resultados lograron observar que durante las mejores

\section{py)eon


experiencias de rendimiento se presentaron mayores niveles de flow y de emociones positivas combinados con menores niveles de ansiedad.

Jiménez-Torres, Godoy-Izquierdo, Ramírez, Navarrón y Godoy (2013) compararon los niveles de flow experimentado en 153 corredores antes, durante y después de una maratón utilizando cuestionario. Obtuvieron como resultado que es la propia actividad deportiva la que actúa en mayor medida como precursora de flow.

Relacionado con lo anterior, Fernández et al. (2015) analizaron si corredores de maratón de distinto nivel de rendimiento experimentaban estados de flow en su práctica; así como también la manera en cómo lo hacían y su posible relación con el rendimiento deportivo. Demostraron con los resultados que los corredores experimentaban episodios de flow con cierta frecuencia o profundidad pero no de la misma manera, así mismo confirmaron que la experimentación de los estados de flow puede ser un beneficio importante en el rendimiento de la competición.

Por otro lado, aparte de investigar la sensación de flow en sí misma, se han investigado múltiples aspectos psicológicos implicados en las carreras y maratones, como la motivación intrínseca. Con respecto a este tema Ruiz-Juan y Zarauz (2014), realizaron un estudio con el fin de analizar la motivación que tenía un grupo de maratonistas para correr. Como resultado obtuvieron que las motivaciones más altas fueron intrínsecas o relacionadas con la tarea, es decir, orientadas hacia la salud y, las más bajas, estaban relacionadas con la motivación extrínseca. 
Sicilia, Moreno y Rojas (2008), investigaron sobre la relación entre perfiles motivacionales y la experiencia de flujo (flow) en estudiantes de educación física. La muestra empleada fueron 983 estudiantes de 14-16 años. Se realizaron cuestionarios para medir los perfiles motivacionales (orientación a la tarea y orientación al ego), la motivación hacia el clima y el flow, agrupando los perfiles en perfiles de alto nivel motivacional (40,28\%), bajo perfil motivacional $(27,57 \%)$ y un perfil de dominio motivacional $(32,15 \%)$, siendo que los distintos perfiles diferían de manera significativa en sus experiencias de flow.

Las anteriores investigaciones representan una muestra del estado de la cuestión sobre el estudio del flow. Ahora bien, existe poca información desarrollada sobre el tema del flow y sus dimensiones en Costa Rica. Por lo anterior, se vuelve imperativo repasar la teoría con la que se cuenta y que le da sustento al presente trabajo de investigación.

Para entender la base del flow se parte de que este estado puede ser experimentado por personas de todas las edades, de ambos sexos y en cualquier cultura, siempre y cuando estén realizando algún tipo de actividad recreativa (Orta-Cantón y Sicilia-Camacho, 2015).

En el caso específico del deporte, los autores Jackson y Marsh (1996) lo definen como “(...)el estado psicológico óptimo para la ejecución. Se produce cuando el deportista se encuentra totalmente conectado con la ejecución que realiza (...) [de modo que se considera como el] "proceso psicológico que subyace al máximo rendimiento" (p. 10). En el ámbito deportivo algunos deportistas describen el estado de flow como "estar en una burbuja", tener satisfacción completa, estar focalizado, estar en la zona, tener un compromiso total, estar

\section{psico


completamente sosegado, estar en marcha, estar concentrado, ser imparable, estar flotando, tener un control total (Orta-Cantón y Sicilia-Camacho, 2015).

El constructo flow o experiencia de flujo como se le suele llamar en español, es un constructo nuevo en la psicología del deporte sin embargo desde que Csikszentmihalyi inició sus estudios en este tema, ha sido un constructo ampliamente estudiado y aplicado en diferentes ámbitos, siendo uno de ellos, la psicología de la felicidad (Csikszentmihalyi, 1996)

En sus descubrimientos para comprender la felicidad, Csikszentmihalyi encontró que la misma no depende de los acontecimientos externos sino de cómo las personas los interpretan. La felicidad es una condición vital que se debe preparar, cultivar y defender individualmente. Si la persona controla su experiencia interna pueden ser capaces de determinar su calidad de vida lo que se acerca a sentir la felicidad. De aquí, se deriva el término de experiencia óptima, que se describe como esos mejores momentos que suceden cuando el cuerpo o la mente de una persona ha llegado al límite de su esfuerzo para conseguir algo difícil de alcanzar y que valiera la pena.

Las experiencias óptimas, añaden un sentimiento de dominio o como menciona el autor, un sentimiento de participación al determinar el contenido de la vida. Estas experiencias dependen de la capacidad por parte del individuo de controlar lo que sucede en su conciencia basándose según su esfuerzo y creatividad. Cuando la persona puede enfrentarse a los desafíos y siente que lo está haciendo bien, entra en un estado tan placentero que su concentración hace que pierda conciencia de sí mismo, liberándose de cualquier 
preocupación y perdiendo la noción del tiempo. Es de esta forma y a partir de esta experiencia óptima que Csikszentmihalyi, elaboró la teoría del flow (Csikszentmihalyi, 1996)

Retomando el concepto del flow, Jackson y Marsh (1996) y Jackson y Csikszentmihalyi (2011), han identificado 9 componentes o dimensiones que corresponden a las sensaciones del flow. A continuación se ofrece una descripción de estos componentes basada en Jackson y Marsh (1996); Jackson y Csikszentmihalyi (2011); López et al. (2007) y Fernández et al. (2015):

1. Equilibrio entre el desafío y la habilidad: debe haber una relación estrecha entre lo que la tarea deportiva demanda y las capacidades de la persona que la realiza.

2. Fusión acción y atención: integración de las capacidades físicas y las mentales para producir un resultado óptimo.

3. Metas claras: deben caracterizarse por ser ajustadas a las necesidades del corredor; ni muy altas ni muy bajas, deben tener el grado de dificultad correcto y estar dirigidas a la meta específica.

4. Feedback o retroalimentación: se trata de la información recibida por el corredor sobre su desempeño en la actividad deportiva. La primera fuente de retroalimentación es el cuerpo, y la segunda son terceras personas involucradas.

5. La concentración en la tarea encomendada: implica una buena cantidad de control sobre el cuerpo, el ambiente y la mente por parte del deportista.

\section{pyes


6. Sensación de control: se relaciona con la anterior en el sentido de que si se genera una sensación de control se puede llegar a percibir como una fortaleza para acabar con la tarea.

7. Pérdida de conciencia del propio ser: se da un proceso en el que el atleta se libera de sus preocupaciones, malestares y pensamientos negativos; se interpreta como una liberación mental.

8. Transformación del tiempo: se da una alteración en la percepción del tiempo, si bien puede ser que se perciba más rápido también puede ser más lento.

9. Experiencia autotélica: conocida como experiencia cumbre, implica una experiencia abrumadora de felicidad, ya que la persona se percibe como dueña de su futuro y realmente capaz de realizar las metas impuestas. Implica que la persona se cree capaz de asumir los retos, tienen autocontrol sobre sus actividades, y las regulan, la experiencia que se vive se percibe como agradable y gratificante.

Cabe mencionar que este estado de flujo se puede experimentar con diferentes intensidades y profundidades o bien en diferentes combinaciones de las dimensiones, lo cual quiere decir que no todas las personas experimentan un estado de flow completo, sino que pueden experimentar al menos uno de los componentes. Un macro flow es cuando el atleta percibe las 9 dimensiones y un micro flow se caracteriza porque sólo experimenta algunas de las dimensiones (López et al., 2007).

Por otro lado, la motivación es un factor que determina el adecuado desarrollo del flow en un individuo (Escartí y Cervello, 1994), algunos autores definen la motivación como 
"un mecanismo psicológico que gobierna la dirección, intensidad y persistencia de la conducta" (Moreno, Villodre, Martínez y Cervelló, 2005). En este marco se podría definir la motivación intrínseca como un compromiso que adquiere una persona con una actividad, ya sea deportiva, recreativa, etc. por el sólo placer y disfrute que ésta le produce.

\section{Método}

Como se mencionó, en esta investigación se indaga en la relación que existe entre la motivación intrínseca y el estado flow, así como respecto a posibles diferencias que se puedan encontrar según la longitud de la carrera de atletismo. Para esto, se encuestó a 63 corredores costarricenses de ambos sexos, en donde 33 fueron mujeres y 30 hombres, lo que constituye un $52.4 \%$ y $47.5 \%$ respectivamente. Las edades oscilan entre los 19 y 64 años. 26 sujetos participaron en distancias menores a 42 kilómetros, lo cual equivale a un $41.3 \%$. Por otro lado, se contó con 37 corredores (un 58.7\%), que participaron en una maratón completa. El cuestionario estaba formado por preguntas demográficas (sexo, edad, distancia de carrera realizada) y se utilizó la escala Flow State Scale (FSS) la cual fue elaborada por Jackson y Marsh (1996), la cual consta de 9 dimensiones del flow, compuesta por 36 ítems, los cuales explican la experiencia que se recuerda de una maratón, las respuestas brindadas son de tipo cerrado, van de 0 (totalmente en desacuerdo) a 10 (totalmente de acuerdo). La confiabiliadad tanto total como por escala para esta investigación fueron altas (Alfa de Cronbach de.70 a .92). Para la motivación intrínseca se utilizó la escala de Motivación en el Deporte (EMD),

\section{psco


la cual fue creada por Briére, Vallerand, Blais y Pelletier en 1995. La misma cuenta con 28 reactivos relacionados con la motivación, las subescalas se evaluaron con 7 puntos en donde el 1 significa "nunca" y 7 corresponde a "siempre", mientras que el 4 se debe a un punto medio que indica “indecisión” (López, 2007).

La información se recolectó enviando un link a los probables participantes, por medio de redes sociales y correo electrónico. Este link dirigía a la encuesta que se digitó por medio de Lime Survey, software de encuesta en línea. Posteriormente se extrajo la base de datos con la información recolectada. Esta base de datos se analizó por medio del SPSS en la versión 21, se realizaron pruebas t para determinar diferencias por sexo y distancia recorrida, así como correlaciones bivariadas en el caso de la relación entre motivación intrínseca y estado de flow.

\section{Resultados}

Se realizó pruebas t de diferencia de medias para identificar diferencias entre las dimensiones del flow según sexo y distancias recorridas. En esta muestra no se encuentran diferencias significativas en torno a hombres y mujeres en el estado del flow y la motivación intrínseca. Se encuentra una diferencia entre la dimensión del flow denominada claridad de objetivos según la distancia recorrida $(\mathrm{T} .014=-2.52 \mathrm{p}=.015)$, siendo que las personas que realizan recorridos de menos de $42 \mathrm{~K}(\mathrm{M}=9.01 \mathrm{DE}=.997)$ son las que puntúan menos frente a los que realizan recorridos de 42K $(\mathrm{M}=9.60 \mathrm{DE}=.771)$. La misma diferencia se encontró 
en la dimensión equilibrio reto-habilidad (T.901=-2.43, p=.018), siendo las personas que realizan recorridos de menos de $42 \mathrm{~K}$ los que puntúan menor $(\mathrm{M}=8,07, \mathrm{DE}=1.33)$ en comparación con los corredores de maratón $(\mathrm{M}=8.37, \mathrm{DE}=1.32)$.

Se encontraron correlaciones significativas entre la motivación intrínseca y algunas de las dimensiones del estado de flow. Refiérase a la figura 1 para detallar estas correlaciones.

Tabla 1. Correlaciones entre dimensiones del flow y Motivación intrínseca

\begin{tabular}{|c|c|c|c|c|c|c|c|c|c|}
\hline & 2 & 3 & 4 & 5 & 6 & 7 & 8 & 9 & 10 \\
\hline 1. Motivación Intrínseca & $.33^{* * *}$ & $.28 *$ & $.31 * *$ & $.33^{* *}$ & $.55 * *$ & $.30 *$ & $.44 * *$ & .21 & $.32 *$ \\
\hline 2. Equilibrio Reto Habilidad & - & $.55 * *$ & $.65 * *$ & $.87 * *$ & $.73 * *$ & $.71 * *$ & $.68 * *$ & .05 & $.69 * *$ \\
\hline 3. Automatismo & & - & $.34 * *$ & $.44 * *$ & $.55^{* *}$ & $.45 * *$ & $.46 * *$ & $.30 *$ & $.31 *$ \\
\hline 4. Claridad Objetivos & & & - & $.73 * *$ & $.59 * *$ & $.55 * *$ & $.57 * *$ & -.11 & $.47 * *$ \\
\hline 5. Claridad Feedback & & & & - & $.66 * *$ & $.80 * *$ & $.70 * *$ & -.07 & $.75 * *$ \\
\hline 6. Concentración & & & & & - & $.64 * *$ & $.73 * *$ & .21 & $.55 * *$ \\
\hline 7. Sentimiento Control & & & & & & - & $.69 * *$ & .019 & $.825 * *$ \\
\hline 8. Pérdida Autoconciencia & & & & & & & - & .09 & $.56^{* *}$ \\
\hline 9. Distorsión Tiempo & & & & & & & & - & .09 \\
\hline 10. Experiencia autotélica & & & & & & & & & - \\
\hline
\end{tabular}

Nota: *Correlaciones significativas al nivel $0.05, * *$ Correlaciones significativas al nivel 0.001 


\section{Discusión}

A raíz de esta investigación se logró comprobar que en 7 componentes de 9 que conforman el estado de flow no hay diferencias significativas en la experimentación del estado de flow en personas que participaron en 42k y en menos de 42k. Sin embargo, sí hay una diferencia significativa en las escalas de equilibrio reto-habilidad y claridad de objetivos. Aunque la diferencia es mínima cabe resaltar que los corredores de maratón puntúan más alto en equilibrio reto-habilidad y en claridad de objetivos.

Con respecto a la dimensión de equilibrio reto-habilidad se entiende que es necesario que haya un equilibrio entre el establecimiento de los retos deportivos y las habilidades del corredor. Para lograr el equilibrio, la meta que se establece debe ser realista y retadora pero también las habilidades y capacidades del deportista deben ser de alto rendimiento (Jackson y Csikszentmihalyi, 2011).

El establecimiento de metas es una condición que los deportistas instauran con anterioridad a la competición, desde los entrenamientos, ya que son la guía para alcanzar metas a corto y mediano plazo (López, et al., 2007). Las metas están dirigidas hacia el desarrollo de las acciones de las tareas, centrándose en cómo un deportista puede superar sus propios límites dejando de lado los resultados de los otros atletas (López et al., 2007). Si bien es cierto para correr cualquier distancia se requiere de un alto rendimiento es importante recalcar que la mayoría de las personas que practican el running en el mundo, suelen preferir distancias entre 5, 10 y 21 kilómetro. Por ejemplo, en el año 2018 hubo 2.9 millones de 
corredores en 5 kilómetros, en 10 kilómetros participaron 1.8 millones y en media maratón 2.1 millones. Con respecto a las maratones, aunque en esta distancia ha aumentado el número de participantes sigue siendo la que menos corredores registra, alcanzando los 1.1 millones. (Jakob, 2019)

Una posible hipótesis que explique estas diferencias es que para correr distancias de $42 \mathrm{~K}$ o más se requiere de un planeamiento anticipado por lo que se debe conocer los retos que una distancia como ésta presenta; y cuáles son las habilidades que el corredor posee para poder responder a las demandas de esta distancia. Según el Dr. Gonzalo Samitier, traumatólogo especialista en lesiones deportivas, entrevistado en El Confidencial, 2018, las personas que se propongan como objetivo participar en una maratón deben comprender que es un reto bastante ambicioso especialmente si no se ha realizado antes. Por ello es necesario que la preparación sea inteligente y planificada en donde la persona sea consciente de sus límites durante el proceso para mantenerse en una zona segura y saludable y así lograr alcanzar el objetivo.

De igual manera, los participantes de una maratón establecen metas a corto y mediano plazo antes de participar en cualquier distancia, ya que por la dificultad se requiere de un entrenamiento previo muy riguroso. A diferencia de los corredores élite, que usualmente su meta final es alcanzar el gane de la carrera, para los demás participantes sus metas están más dirigidas hacia superar sus propios límites, ya sea superando su propia marca o inclusive superando sus propias barreras emocionales. En un estudio realizado por Johnsgard (1989) 
citado en Breheny (2001), comparó los resultados obtenidos sobre los motivos de correr una maratón entre 150 mujeres corredoras recreativas versus 10 corredoras elite. Entre los resultados obtuvo que en ambos grupos se encontraba el cumplir con el reto sin embargo en el grupo de las corredoras elite predominaban los motivos de competición, fama, fortuna e identidad.

Se comprobó que para esta muestra no hay diferencias significativas sobre la experiencia del flow y la motivación intrínseca con respecto al sexo. Esto quiere decir que tanto hombres como mujeres perciben el estado de flow durante la práctica deportiva. Hay que recordar que el flow es un estado que se experimenta cuando se realiza alguna actividad recreativa que produce placer y que lo pueden experimentar todas las personas independientemente de su edad, género o cultura (Orta-Cantón y Sicilia-Camacho, 2015), y que tanto los corredores como las corredoras que realizan esta actividad deportiva lo hacen sobre todo para satisfacer su motivación intrínseca.

Por último, la correlación entre la motivación intrínseca y la mayoría de las dimensiones del flow, fue hallada previamente en el estudio de Moreno et al. (2005) donde se estipuló que las personas que tienen mayor motivación intrínseca tienen mayor probabilidad de experimentar el flow. Como se mencionó las relaciones positivas entre la motivación y las dimensiones del flow implican que debe haber un equilibrio entre el reto que trae consigo la competición y la habilidad del corredor; esto se relaciona también con tener claridad en los objetivos y la retroalimentación. Es decir, que la preparación previa 
entre entrenadores o corredores es vital para desarrollar el conocimiento en las propias habilidades y balancear las demandas que serán impuestas. Aunado a lo anterior, las correlaciones positivas con el autocontrol y la experiencia autotélica indican que la motivación está relacionada con la experiencia de sentirse capaz y en control de realizar la actividad, lo que va a conducir a una percepción de autoconfianza y felicidad.

Por otro lado, no se encontró una relación significativa entre la motivación intrínseca y la distorsión del tiempo, la cual es una de las dimensiones del estado de flow. Esto se podría deber a que tanto para los maratonistas como para las personas que corren distancias menores, no es motivante estar pensando en cuanto tiempo van a terminar la práctica deportiva. Algunos deportistas evaluaban su motivación para participar en un encuentro deportivo no en función de los resultados de la competición sino en función de su actitud hacia la misma, o sea el esfuerzo o la mejora personal que ésta le podría llegar a dar (Cecchini, González, Carmona y Contreras, 2004).

En cuanto aspectos limitantes de la investigación, en primer lugar el cuestionario al ser por medio digital, no se administró inmediatamente después de terminar la competición por lo que algunas preguntas resultaban más complejas para los participantes. Se recomienda también, aumentar la muestra para capturar una gama más amplia de experiencias. Relacionado con la anterior, en Costa Rica las maratones son escasas, por lo que un estudio que desee investigar estos procesos con maratonistas debe considerar el factor de tiempo para la recolección de datos. Estas limitantes se pueden mejorar para futuras investigaciones en el

\section{pesco


campo, como por ejemplo, aún queda sin conocerse la relación entre el tipo de atleta con las variables estudiadas, o bien el tipo de deporte.

Se comprobó por medio de esta investigación que existe una relación entre algunas de las dimensiones del flow y la motivación intrínseca. No se presentaron diferencias por sexo. La única diferencia se dio entre dos dimensiones del flow (equilibrio reto-habilidad y claridad de metas) en cuanto a personas que corren menos de $42 \mathrm{~K}$ y las que corren la maratón. Esta diferencia podría explicarse por los años de experiencia y por los requerimientos que tiene cada carrera en sí, siendo las más largas, las que exigen mayor preparación y autoconocimiento.

\section{Referencias}

Breheny, K., (2001). Motives for running a marathon: The contribution of Achievement Motivation (Tesis inédita de doctorado). Fordham University, Nueva York. Recuperado de https://search.proquest.com/docview/305574967

Cecchini, A., González, C., Carmona, M. y Contreras, O. (2004). Relaciones entre clima motivacional, la orientación de meta, la motivación intrínseca, la auto-confianza, la ansiedad y el estado de ánimo en jóvenes deportistas. Psicothema, 16(1) 104-10. Recuperado de http://www.psicothema.com/pdf/1168.pdf 
Csikszentmihalyi, M. (1996). Fluir. Una Psicología de la Felicidad. España: Editorial Kairós.

Escartí, A. y Cervello E. (1994). La motivación en el deporte. Entrenamiento psicológico en el deporte: Principio y aplicaciones. Valencia: Albatros educación.

Fernández, M., Godoy-Izquierdo, D., Jaenes, J.C., Bohórquez, M., y Vélez, M. (2015). Flow y rendimiento en corredores de maratón. Revista de Psicología del Deporte, 24(1), 919. Recuperado de https://www.redalyc.org/articulo.oa?id=235139639002

Jackson, S. y Csikszentmihalyi, M. (2011). Fluir en el deporte. España: Editorial Paidotribo.

Jackson, S.A. y Marsh, H.W. (1996). Development and validation of a scale to measure optimal experience: the flow state scale. Journal of Sport \& Exercise Psychology, (18), 17-35. Recuperado de https://pdfs.semanticscholar.org/553c/9421dbb9d23735b0936e3743eaa021f0b4f4.p df

Jakob, J. (2019). The State of Running 2019. [Por RunRepeat.com and the International Association of Athletics Federations]. Recuperado de https://runrepeat.com/state-ofrunning

Jiménez-Torres, M., Godoy-Izquierdo, D., Ramírez, M.J., Navarrón, E. y Godoy, J.F. (2013). Flow experimentado por atletas de maratón antes, durante y después de la

\section{pyseor


competición. Anuario de Psicología Clínica y de la Salud, 9, 85-87. Recuperado de https://dialnet.unirioja.es/servlet/articulo?codigo $=4671017$

Koehn, S., Donald, B., y Paramei, G. (2018). Antecedents of Flow and the Flow-Performance Relationship in Cricket. Kinesiology, 50(2), 277-284. https://doiorg.ezproxy.sibdi.ucr.ac.cr/10.26582/k.50.2.6

La Nación. (30 de mayo, 2011). Crece en el país la pasión por el atletismo aficionado. La Nación. Recuperado de: https://www.nacion.com/puro-deporte/otros-deportes/creceen-el-pais-la-pasion-por-el-atletismoaficionado/TYYCHPZZ2BCJ7LLEXJ5NY2SPLY/story/

López, M., Torregrosa, M. y Roca, J. (2007). Características del "flow”, ansiedad y estado emocional, en relación con el rendimiento de deportistas de élite. Cuaderno de psicología del deporte, 7(1), 25-44. Recuperado de Recuperado de https://revistas.um.es/cpd/article/view/54641

Moreno, J., Villodre, N., Martínez, C. y Cervelló, E. (2005). Motivación, disciplina, coeducación y estado de flow en educación física: Diferencias según la satisfacción, la práctica deportiva y la frecuencia de práctica. Cuadernos de Psicología del Deporte, 5(1-2), 225-243. Recuperado de: https://www.um.es/univefd/motiflow.pdf

Orta-Cantón, A. y Sicilia-Camacho, A. (2015). Investigando los momentos óptimos en el deporte: una revisión del constructo flow. Revista Brasileira de Ciencias del Deporte, 37(1), 96-103. DOI: 10.1016/j.rbce.2014.01.001 
Ruiz-Juan, F. y Zarauz, A. (2014). Análisis de la motivación en corredores de maratón Españoles. Revista Latinoamericana de Psicología, 46(1), 1-11.

Samitier, G. (2018). Siete claves para correr una maratón sin poner en riesgo tu salud. El Confidencial. Recuperado de: https://www.elconfidencial.com/alma-corazonvida/running/2018-04-05/preparar-maraton-objetivo-estrategia-bra_1544266/

Sicilia, A., Moreno, J.A. y Rojas, A.J. (2008). Motivational Profiles and Flow in Physical Education Lessons. Perceptual and Motors Skills, 106, 473-494. 